\section{Comments on "Interference From 24-GHz Automotive Radars to Passive Microwave Earth Remote Sensing Satellites"}

Yann H. Kerr, Guy Rochard, Phillippe Tristant, Steve English, Markus Dreis, Ad Stoffelen, Jean Pla, Björn Rommen, Edoardo Marelli, Klaus Ruf, and Peter Bauer

\begin{abstract}
In a recent paper, Younis et al. propose an apparently interesting methodology for the computation of the interference received by a spaceborne passive sensor from terrestrial interferences. However, the paper seems to require some clarifications, as some of the conclusions are questionable. This comments paper aims at identifying the most outstanding issues of the publication.
\end{abstract}

Index Terms-Automotive radar, compatibility, Earth Exploration Satellite Sensors (EESS), radiometry.

\section{INTRODUCTION}

In a recent paper [1], Younis et al. propose an interesting methodology for the computation of the interference received by a spaceborne passive sensor from terrestrial interferences. However, it appeared that the publication was questionable on several aspects as it raised a number of questions which require to be reviewed. It was also felt that, in some instances the assumptions made are erroneous. Consequently, some of the conclusions of the publication might be misleading, and thus [1] requires some clarifications.

\section{MAIN ISSUES}

So as to give a basis for clarifying potential issues with [1] and avoid misleading conclusions here are a few points which have to be stressed.

1) In [1], two basic notions for earth exploration satellite sensors (i.e., sensitivity and interference threshold) are confused. The exact signification of these two terms is as follows.

Manuscript received January 18, 2005.

Y. H. Kerr is with the Centre d Etudes Spatiales de la Biosphère (CESBIO), 31401 Toulouse Cedex 9, France (e-mail: Yann.Kerr@ cesbio.cnes.fr).

G. Rochard is with the Centre de Météorologie Spatiale, Météo-France, 22307 Lannion, France and also with the International TOVS Working Group (e-mail: guy.rochard@meteo.fr).

P. Tristant is with Météo-France, 31057 Toulouse, Cedex France and also with the European Meteorological Network (EUMETNET) (e-mail: philippe.tristant@meteo.fr).

S. English is with the Met Office, Devon EX1 3PB U.K. (e-mail: stephen.english@metoffice.gov.uk).

M. Dreis is with Eumetstat, D-64295 Darmstadt, Germany (e-mail: dreis@eumetsat.de)

A. Stoffelen is with the Koninklijk Nederlands Meteorologisch Instituut (KNMI), 3730 AE De Bilt, The Netherlands (e-mail: Ad.stoffelen@knmi.nl).

J. Pla is with the Centre National d Etudes Spatiales (CNES), 31401 Toulouse, Cedex 9 France (e-mail: jean.pla@cnes.fr).

B. Rommen and E. Marelli are with the European Space Research and Technology Centre, European Space Agency (ESTEC/ESA), 2200AG Noordwijk,

The Netherlands (e-mail: bjorn.rommen@esa.int; edoardo.marelli@esa.int)

K. Ruf is with the German Aerospace Center (DLR), D-53227 Bonn, Germany (e-mail: klaus.ruf@dlr.de).

P. Bauer is with the European Centre for Medium-range Weather Forecasting (ECMWF), Reading RG2 9AX, U.K. (e-mail: peter.bauer@ecmwf.int).

Digital Object Identifier 10.1109/TGRS.2005.846449 a) The sensitivity reflects the minimum variation in radiance that a given sensor is able to detect. The word sensitivity is reserved to the satellite resolution itself, which is the performance actually achieved by the satellite. Recommendation ITU-R (International Telecommunication Union Radiocommunication sector) SA.1028-2 indicates the list of the applicable sensitivities for each frequency band.

b) Concerning the interference threshold, it is quite easy to realize that if interference is allowed at the sensitivity level, you will no longer be able to detect variations in radiance with this sensitivity. To that respect, Recommendation ITU-R SA.1029-2 shows that the interference threshold must be taken as $20 \%$ of the sensitivity.

2) In [1], the authors use the $0.3-\mathrm{K}$ sensitivity as an interference threshold while the current ITU-R Recommendation SA.1028-2 gives $0.05 \mathrm{~K}$ and, as stated in 1) above, Recommendation SA.1029-2 gives the corresponding interference threshold at $0.01 \mathrm{~K}$. This represents a difference of $15 \mathrm{~dB}$ in interference calculation, which is certainly not negligible. The ITU-R group responsible for all ultrawideband issues [Task Group (TG) 1/8], including short-range radars (SRRs), has definitively recognized the adequacy and relevance of Recommendation ITU-R SA.1029-2. It is to be noted the that last revision of this Recommendation (SA.1029-2) has been agreed and proposed by meteorological experts from many countries and institutes.

3) In [2], English compares the interference threshold for 23.6-24.0 GHz over land and sea and found them to be similar. When radio-frequency interferences (RFIs) are greater than 1/10th of the innovation it is considered to be significant (i.e. likely to cause misinterpretation of the innovation). ${ }^{1}$ It was studied how often at a given threshold (say $0.2 \mathrm{~K}$ ) 1/10th of the innovation is less than the threshold. For sea points $32 \%$ of points fall below $0.2 \mathrm{~K}$. That is to say RFI of $0.20 \mathrm{~K}$ would exceed $10 \%$ of the innovation $32 \%$ of the time. For land points [2] shows that RFI tolerance is slightly lower than for sea points, not higher as might have been expected. $38 \%$ of land points fall below $0.20 \mathrm{~K}$. That is to say RFI of $0.20 \mathrm{~K}$ would exceed $10 \%$ of the innovation vector $38 \%$ of the time. To reduce significant interference to occur less than $5 \%$ of the time over land would require a threshold of $0.03 \mathrm{~K}$ at most, preferably lower. This is broadly consistent with Recommendation ITU-R SA.1029-2. It has also to be emphasized that these criteria are more than likely to become even more stringent in the future while passive sensors will improve, taking advantage of the technological advances, to better meet accuracy and sensitivity requirements.

4) Reference quoted in [1] under label [5] ([3] of this correspondence) is inadequate and should be ITU Radio Regulations footnote 5.340, which states that "all emissions are prohibited" in the 23.6-24-GHz band, among others.

5) The generic formula given in [1] under number (8) and which is based on the development given in the appendix might be of interest to perform generic calculations independently of satellites characteristics. However, during recent ITU-R meetings [Working Party (WP)

\footnotetext{
${ }^{1}$ The innovation is the difference between what is actually measured and what is expected to be measured based on existing knowledge of the atmospheric state (for numerical weather preduction short-range forecast).
} 
7C and TG 1/8], this formula has been shown as being a priori correct for calculation performed for cross-track nadir instruments but not for conically scanning instruments which do not have zenith incident angle with the earth. This fact makes the pixel area larger and reduces the spectral density corresponding to the interference threshold. This generic formula should thus include an additional $\cos (\theta)$ factor and be consequently of the form

$$
n=\frac{4 \pi}{P C} \frac{k \delta T_{\mathrm{th}}}{\lambda^{2}} \cos (\theta) e^{\tau / \cos (\theta)}
$$

where

$n \quad$ number of SRR per unit area (square kilometers);

$P \quad$ effective isotropic radiated power (EIRP) density of a SRR (including bumper loss);

$\delta T_{\text {th }} \quad$ allowable interference treshold (in kelvin);

$C \quad$ coupling factor (direct path and scattering effect) on a polarized basis;

$\tau \quad$ atmospheric opacity from the surface to the satellite;

$\theta \quad$ incidence angle of the satellite beam from nadir;

$\lambda \quad$ wavelength (in meters);

$k \quad$ Boltzmann's constant $\left(1.3810^{-23} \mathrm{~J} / \mathrm{K}\right)$.

6) The value for the opacity which is used $(\tau=0.5)$ is not adequate for the 23.6-24-GHz band. For example, at an angle $\theta$ of $0^{\circ}$ (i.e., at zenith), it gives an atmospheric attenuation of $2.17 \mathrm{~dB}$, when the maximum is of $1 \mathrm{~dB}$ (in tropical zones) and a typical range is from 0.5-0.8 dB, according to Recommendation ITU-R P.676-5. This means that $\tau=0.15$ is a better suited figure.

7) The measurement sketch described in Section IV of [1] introduces an inconsistency with regard to the geometry of the interference at the satellite. This is due to the fact that the distances in the anechoic chamber in Ispra $(20 \mathrm{~m})$ are not comparable to those experienced with a satellite (more than $700 \mathrm{~km}$ ). As shown in Fig. 3 of [1], it implies that the measurements combine the direct path and the scattered path at different incidence angles whereas in real life the two paths are parallel. Acknowledging that the direct path interference is controlled by the antenna sidelobe attenuation, it would have an impact on the combined interference. In addition, this discrepancy may also introduce a difference in phase that could influence the measurements. It is difficult to accurately assess the impact of this on the validity of the results but the issue should have been discussed in the paper, as it makes interpretation of the results difficult. However, the measurements and simulations contained in [1] seem to show that the scattered effect is much more important at low elevation. As an example, based on Fig. 3 of [1], and assuming that the path of the scattered interference is parallel to the direct path, this would result in a higher combined interference and hence a lower coupling factor.

8) The paper [1] suggests a value of $-26 \mathrm{~dB}$ for the coupling factor but does not provide real rationale for it. Table IV in [1] gives mean and max values of the coupling factor, but it is averaged over all angles. On the other hand, Fig. 11 in [1] provides "average polarimetric coupling coefficient over $\theta$ " that shows that at $\theta=0^{\circ}$ (which corresponds to the zenith and hence to the elevation related to cross-track nadir sensors), the average coupling is roughly $-22 \mathrm{~dB}$ for vertical polarization and $-15 \mathrm{~dB}$ for horizontal polarization. As average values, this would support to retain $-15 \mathrm{~dB}$ as coupling factor in the final calculations or at $a$ minimum $-22 d B$ if we only account for vertical polarization. A coupling factor of $-26 \mathrm{~dB}$ is hence too low to be suggested as typical. Current and agreed calculation within ITU-R or Europe have considered a -24-dB coupling factor, based on United States National Oceanic and Atmospheric Administration (NOAA) simulations and tests, that is hence already lower than what the results seem to show in [1] . For global (worldwide) numerical weather prediction (NWP) as for climatology, it is necessary to have the same accuracy for the satellite radiances over lands and over oceans in the band 23.6-24 GHz. For local and regional NWP as over Europe, the U.S., China, etc., most of the data needed are over land and the progress of these NWP requests more and more accurate data. That includes now casting to protect people and goods.

It is thus very important to ensure data quality and minimize potential sources of interferences. It was felt that [1] started the right approach through the analysis of potential level of interferences caused by the implementation of a new system. It was nevertheless found most unfortunate that some of the assumptions made were not always the best and that some aspects of the methodology potentially lead to underestimations of the actual interference level. It is therefore important to correct misleading conclusions from [1] so as to avoid wrong decision, jeopardizing the future or NWP.

\section{REFERENCES}

[1] M. Younis, J. Maurer, J. Fortuny-Guasch, R. Schneider, W. Wiesbeck, and A. J. Gasiewski, "Interference from 24-GHz automotive radars to passive microwave earth remote sensing satellites," IEEE Trans. Geosci. Remote Sens., vol. 42, no. 7, pp. 1387-1398, Jul. 2004.

[2] S. J. English, "Assessment of the requirement for 23.6-24.0 GHz observations for weather forecasting," Met Office, Devon, U.K., Forecasting Res. Tech. Rep. 440. Available from www.metoffice.gov.uk, 2004.

[3] ITU, "Footnote 880. International Telecommunication Union Radio regulation Std.," Int. Telecommun. Union, Geneva, Switzerland, 1988.

\section{Reply to Comments on "Interference From 24-GHz Automotive Radars to Passive Microwave Earth Remote Sensing Satellites"}

\author{
A. J. Gasiewski, W. Wiesbeck, and M. Younis
}

We appreciate that authors Kerr et al. concur that our paper [1] provides the right approach to the analysis of potential interference from anthropogenic sources to remote sensing satellites. The potential for such interference is likely to grow as new active systems are developed, necessitating acceptable procedures for interference analysis based on accepted scientific knowledge and engineering principles. While some simple clarifications are in order to improve the acceptability of our procedure [cf. points 1) and 5)], we suggest, however, that Kerr et al.

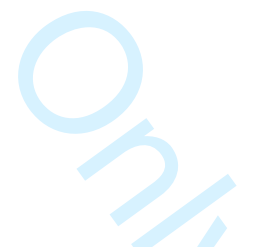

Digital Object Identifier 10.1109/TGRS.2005.846447 